\title{
Coronary Plaque Erosion after Abemaciclib Treatment Onset: An Unknown Side Effect?
}

\author{
Alberto Vera ${ }^{1}$ Fernando Rivero ${ }^{1}$ Jorge Salamanca ${ }^{1}$ Teresa Alvarado-Casas ${ }^{1}$ Fernando Alfonso ${ }^{1}$ \\ 1 Instituto de Investigación Sanitaria (IIS-IP), Hospital Universitario de \\ la Princesa, CIBERCV, Madrid, Spain \\ Thromb Haemost 2021;121:976-978.

\begin{abstract}
Address for correspondence Fernando Alfonso, MD, PhD, Cardiology Department, Instituto de Investigación Sanitaria IIS-IP, Hospital Universitario de La Princesa, Universidad Autónoma de Madrid, CIBERCV. C/ Diego de León 62, Madrid 28006, Spain (e-mail: falf@hotmail.com).
\end{abstract}

Abstract
Keywords
- thrombosis
- acute myocardial
infarction
- cardiology

The current article describes a 72-year-old woman who suffered an acute myocardial infarction due to plaque erosion (PE) 2 weeks after abemaciclib treatment onset due to advanced breast cancer. Abemaciclib is a cyclin-dependent kinase 4 and 6 inhibitor that has recently demonstrated efficacy and safety in advanced breast cancer. Of major concern, however, reported thromboembolic rates in randomized clinical trials testing this drug range from 0.6 to $5 \%$. To the best of our knowledge this is the first thrombotic coronary side effect ever reported. We suggest that a treatment that increases thromboembolic risk, such abemaciclib, may have triggered $\mathrm{PE}$ in our patient, 15 days after abemaciclib initiation. New molecules are promising in cancer treatment; however, care must be paid to their potential cardiotoxic effects.
A 72-year-old woman without cardiovascular risk factors was seen in the emergency department for chest pain. Due to advanced breast cancer she had initiated abemaciclib treatment 2 weeks prior to her presentation. The electrocardiogram showed ST-segment elevation in V2-V5. Emergent coronary angiography revealed a mild stenosis in the proximal left anterior descending (LAD) coronary artery with normal coronary flow (-Fig. 1A). Optical coherence tomography of the proximal LAD stenosis revealed a large mixed (red and white) thrombus with some posterior shadowing, but with no signs of plaque rupture, and a good residual lumen, suggestive of plaque erosion (PE) ( $\mathbf{- F i g . ~ 1 B , C ) . ~ I m m e d i a t e l y ~ p r o x i m a l ~ t o ~ t h i s ~ s e g - ~}$ ment, a large plaque with lipid content and infiltrated (bright speckling) neointima, was also detected ( - Fig. 1D). Conservative management with aspirin, ticagrelor, and enoxaparin was decided. Troponin $\mathrm{T}$ peak was $512 \mathrm{ng} / \mathrm{L}$ (normal $<28 \mathrm{ng} / \mathrm{L}$ ). Clinical course was favorable. The patient was discharged on dual antiplatelet therapy.

PE has been described as the underlying pathological substrate in up to one-third of patients with acute myocardial infarction. Advances in intracoronary imaging have improved anatomical characterization of PE in the clinical setting. ${ }^{1,2}$ In contrast with plaque rupture, the pathophysiological mechanisms leading to PE remain poorly understood. ${ }^{1}$ Although female gender and smoking have been linked as risk factors of $\mathrm{PE}{ }^{1}$ other predisposing factors and triggers remain unknown. Abemaciclib is a cyclin-dependent kinase (CDK) 4 and 6 inhibitor that has recently demonstrated efficacy and safety in advanced breast cancer. Of major concern, however, reported thromboembolic rates in randomized clinical trials testing this drug range from 0.6 to $5 \%$. These may significantly underestimate actual thromboembolic complication rates observed in real-world patients. ${ }^{3}$ Furthermore, out of the reported cases of adverse reaction to CDK4/6 inhibitors between years 2018 and 2019, 2.9\% were cardiotoxicities. ${ }^{4}$ The pathophysiological basis for abemaciclib embolic risk and cardiotoxicity is not clearly understood. We suggest that a treatment that increases thromboembolic risk, such abemaciclib, may have triggered PE in our patient, 15 days after abemaciclib initiation. Alternatively, this drug may enhance the thrombotic component occurring during the acute phase of PE explaining the clinical presentation as an ST-segment elevation acute myocardial infarction. ${ }^{5}$ This case, despite its isolated nature, strikingly illustrates that although new

\section{received}

November 7, 2020

accepted after revision

November 27, 2020

published online

December 1, 2020 (c) 2020. Thieme. All rights reserved. Georg Thieme Verlag KG,

Rüdigerstraße 14,

70469 Stuttgart, Germany
DOI https://doi.org/ 10.1055/a-1326-7028. ISSN 0340-6245. 


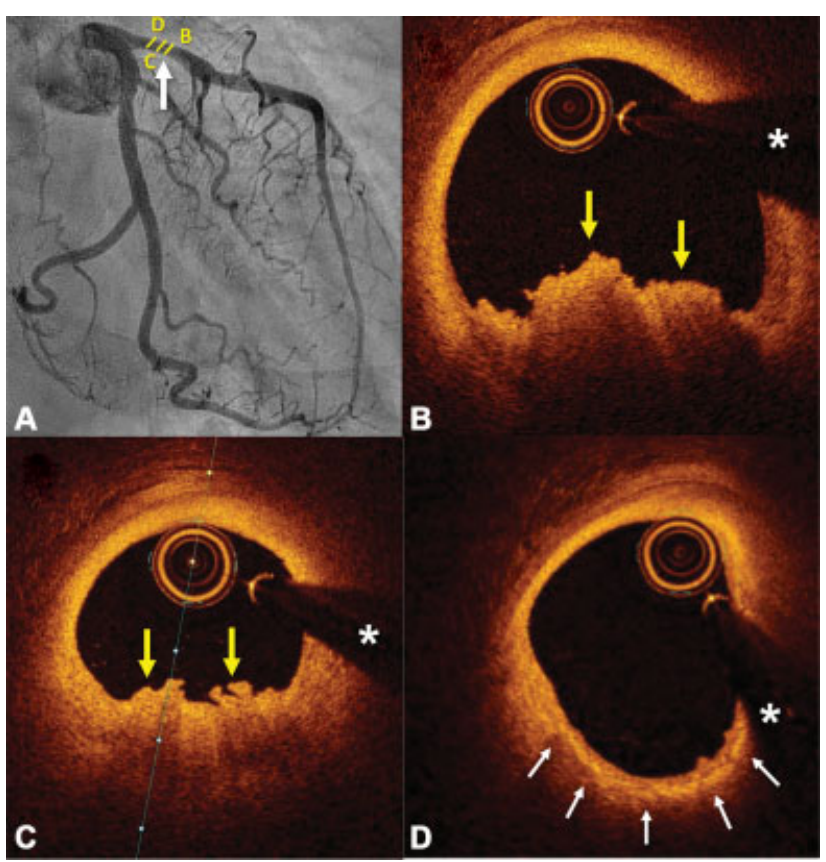

Fig. 1 (A) Coronary angiogram showing a mild stenosis (white arrow) on the proximal left anterior descending coronary artery (LAD). (B-C) Characteristic images suggestive of plaque erosion with some overlying thrombus (yellow arrows). (D) Immediately adjacent proximal plaque with a large lipid content and an infiltrated (bright speckling) neointima (white small arrows). ${ }^{*}$ Denotes wire artifact. molecules are promising in cancer treatment, care must be paid to clarify and monitor their potential cardiotoxic and prothrombotic effects.

\section{Conflict of Interest}

None declared.

\section{References}

1 White SJ, Newby AC, Johnson TW. Endothelial erosion of plaques as a substrate for coronary thrombosis. Thromb Haemost 2016; 115(03):509-519

2 Jia $\mathrm{H}$, Abtahian F, Aguirre AD, et al. In vivo diagnosis of plaque erosion and calcified nodule in patients with acute coronary syndrome by intravascular optical coherence tomography. J Am Coll Cardiol 2013;62(19):1748-1758

3 Gervaso L, Montero AJ, Jia X, Khorana AA. Venous thromboembolism in breast cancer patients receiving cyclin-dependent kinase inhibitors. J Thromb Haemost 2020;18(01):162-168

4 Master SR. Cardiac complications of CDK4/6 inhibitors for breast cancer. J Clin Oncol 2020;38(15):13038

5 Alfonso F, Rivero F. Antithrombotic therapy alone for plaque erosion: ready for a paradigm shift in acute coronary syndromes? Circ Cardiovasc Interv 2017;10(12):e006143 


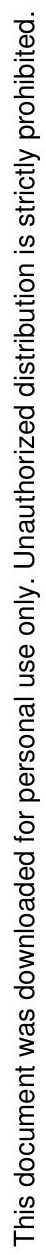

